https://helda.helsinki.fi

\title{
TKTL1 as a Prognostic Marker in Pancreatic Ductal Adenocarcinoma and Its Correlation with FDG-PET-CT
}

\section{Ahopelto, Kaisa}

2021-02

Ahopelto , K, Saukkonen , K, Hagström , J , Kauhanen , S , Seppänen , H, Böckelman , C \& Haglund, C 2021, ' TKTL1 as a Prognostic Marker in Pancreatic Ductal Adenocarcinoma and Its Correlation with FDG-PET-CT ' , Oncology , vol. 99 , no. 3 , pp. 177-185 . https://doi.org/10.1159/000510862

http://hdl.handle.net/10138/340116

https://doi.org/10.1159/000510862

unspecified

acceptedVersion

Downloaded from Helda, University of Helsinki institutional repository.

This is an electronic reprint of the original article.

This reprint may differ from the original in pagination and typographic detail.

Please cite the original version. 


\section{Oncology}

\begin{tabular}{|l|l|}
\hline Manuscript: & OCL-0-0-0 \\
\hline Title: & $\begin{array}{l}\text { TKTL1 as a prognostic marker in pancreatic ductal adenocarcinoma } \\
\text { and its correlation to FDG-PET-CT }\end{array}$ \\
\hline Authors(s): & $\begin{array}{l}\text { Kaisa Ahopelto (Corresponding Author), Kapo Saukkonen } \\
\text { (Coauthor), Jaana Hagström (Co-author), Saila Kauhanen (Co- } \\
\text { author), Hanna Seppänen (Co-author), Camilla Böckelman (Co- } \\
\text { author), Caj Haglund (Co-author) }\end{array}$ \\
\hline Keywords: & Pancreatic cancer, PDAC, PET-CT, TKTL1, Tumor marker \\
\hline Type: & Research Article \\
\hline
\end{tabular}




\section{TKTL1 as a prognostic marker in pancreatic ductal adenocarcinoma and its correlation to FDG-PET-CT}

Kaisa Ahopelto a,b, Kapo Saukkonenª, Jaana Hagström ${ }^{\text {b,c }}$, Saila Kauhanen ${ }^{\text {d }}$, Hanna Seppänenª, ${ }^{*}$ Camilla Böckelman a,b , *aj Haglunda,b

aDepartment of Gastrointestinal Surgery, University of Helsinki and Helsinki University Hospital, Helsinki, Finland bTranslational Cancer Medicine Research Program, Faculty of Medicine, University of Helsinki, Helsinki, Finland cDepartment of Pathology and Oral Pathology, University of Helsinki and Helsinki University Hospital, Helsinki, Finland dDivision of Digestive Surgery and Urology, Turku University Hospital, Turku, Finland *shared last authorship

Short title: TKTL1 in PDAC and its correlation to FDG-PET-CT

Corresponding author:

Kaisa Ahopelto, MD

Department of Surgery

University of Helsinki and Helsinki University Hospital

PO BOX 440

00029 HUS

FINLAND

Phone: +3589471 72426

Email: kaisa.ahopelto@hus.fi

Number of Tables: 3

Number of Figures: 3

Word count: 2684 
KEYWORDS: TKTL1; PDAC; pancreatic cancer; tumor marker; PET-CT

\begin{abstract}
INTRODUCTION: Glucose metabolism in cancer cells differs from noncancerous cells. The expression of transketolase-like protein 1 (TKTL1), a key enzyme in the glucose metabolism of cancer cells, predicts poor prognosis in several cancer types. We studied TKTL1 as a prognostic tool and whether TKTL1 expression correlates with 18F-FDG-PET-TT among patients with pancreatic ductal adenocarcinoma (PDAC).

METHODS: This retrospective study examined two PDAC patient cohorts: 168 patients operated on at Helsinki University Hospital between 2001 and 2011, and 20 patients with FDG-PET-CT results available from the Auria Biobank. We used immunohistochemistry (IHC) for TKTL1 expression, combining results with clinicopathological data.

RESULTS: Five-year disease-specific survival (DSS) was slightly but not significantly better in patients with a high versus a low TKTL1 expression, with DSS of $28.0 \%$ vs $17.3 \%$, respectively $(p=0.123)$. TKTL1 served as a marker of a better prognosis in patients over 65 years old $(p=$ $0.012)$ and among those with TNM class M1 $(p=0.018)$, stage IV disease $(p=0.027)$, or perivascular invasion $(\mathrm{p}=0.008)$.
\end{abstract}

CONCLUSIONS: Our study shows that TKTL1 cannot be used as a prognostic factor in PDAC with the exception of elderly patients and those with advanced disease. The correlation of TKTL1 with 18F-FDG-PET-CT requires further study in a larger patient cohort. (210 words) INTRODUCTION

Globally, nearly half a million $(458,918)$ new cases of pancreatic cancer occur annually resulting in nearly as many deaths $(432,242)$, the incidence of which is rising [1]. While cancer prognosis in recent decades has generally improved, that for pancreatic cancer has not. Many patients present with advanced disease upon diagnosis, whereby only a minority are eligible for curative surgery. Perioperative chemotherapy is offered to patients with locally 
advanced disease, and trials remain ongoing to determine whether all patients might benefit from neoadjuvant chemotherapy [2]. Chemoradiotherapy pre operatively compared to surgery alone offers no overall survival benefit, but leads to a larger rate of R0 resection in patients with borderline disease [3].

In cancer cells, glucose metabolism increases compared to that in normal cells. This offers a novel target for chemotherapy but can also be used to diagnose and monitor tumor growth with positron emission tomography (PET). Nobel laureate Otto Warburg described an increased glycolysis in an aerobic environment via the pentose phosphate pathway (PPP), a typical attribute of cancer cells [4]. Transketolases control the nonoxidative part of PPP. Transketolase-like protein 1 (TKTL1), represents one transketolase isoform that causes rapid tumor-cell growth, while also leading to reduced glucose consumption when suppressed [5]. TKTL1 expression is elevated in a number of cancers, and serves as a marker of poor prognosis in colorectal and gastric cancer among many others [6-16].

18F-fluorodeoxyglucose (FDG), a glucose analog and a marker for glucose uptake in the tissue, is used to diagnose and monitor cancer growth through PET. In pancreatic cancer, $18 \mathrm{~F}$ FDGPET-CT is effective in detecting malignant masses of the pancreas, and superior in detecting lymph node metastases when compared to ultrasound and computed tomography (CT) [17].

In a cohort of 56 pancreatic cancer patients without suspected metastasis in other preoperative imaging, PET-CT identified metastases in the liver, lymph nodes, and bone in 9 patients, ultimately altering the treatment plan [18]. The advantages of PET-CT appear in the detection of distant metastasis. But, when differentiating inflammatory lymph nodes from 
metastatic nodes or determining cancer growth around critical arteries and veins, PET-CT has not proved sufficiently sensitive nor specific [19,20]. PET-CT is, however, valuable in detecting recurrent pancreatic cancer with a $96 \%$ detection rate compared to a $39 \%$ detection rate for CT or magnetic resonance imaging (MRI) [21]. In addition, immunostaining with Ki-67 has been compared to the FDG uptake of pancreatic tumors in PET-CT, but no correlation was found between the proliferation index and FDG uptake [22]. To our knowledge, no tumor markers have been compared to the FDG activity in PET-CT. Yet, a high compared to low FDG uptake represents an independent predictor of survival in pancreatic cancer $[23,24]$. The National Comprehensive Cancer Network (NCCN) guidelines from 2017 do not recommend PET-CT in the preoperative setting [25]. In the updated guidelines from 2019, imaging was not discussed at all [26] nor did it appear in the European Society for Medical Oncology (ESMO) guidelines from 2015. In ESMO's updated version of the guidelines in 2019 (ePub), only new chemotherapy recommendations were listed [27].

The tumor marker currently used to monitor the course of PDAC disease is CA19-9, despite proving less useful during the initial or primary diagnosis [27]. Other serum biomarkers examined are not yet used in clinical practice. Quite modest steps have been taken to personalize treatment among pancreatic cancer patients, only a few mutations were identified as targets for chemotherapy treatment. We still need better biomarkers to evaluate disease progression and prognosis among PDAC patients and to predict the treatment response for chemotherapy regimens. 
Therefore, this study aims to determine whether TKTL1 could serve as a prognostic biomarker in pancreatic cancer and to evaluate the correlation between TKTL1 expression and 18F-PET-CT findings.

\section{MATERIALS AND METHODS}

\section{Patients and tissues}

This study comprised 168 pancreatic cancer patients surgically treated in the Department of Surgery at Helsinki University Hospital between 2001 and 2011. For 166 patients, TKTL1 expression could be determined from tumor tissue microarray (TMA) slides. Among these, 93 (55.4\%) were men, and $80(47.6 \%)$ were older than 65 years. The tumor was located at the head of the pancreas in 124 patients (73.8\%), the tail in $9(5.4 \%)$, the body in $11(6.5 \%)$, and the entire pancreas in one patient $(0.6 \%)$. No patient received neoadjuvant therapy (data missing for $15.5 \%$ of patients), and 73 (43.5\%) patients received postoperative adjuvant chemotherapy. Disease-specific survival (DSS) at 5 years for all patients was 21.3\% [95\% confidence interval (CI) 14.6-28.0]. The median follow-up time was 1.96 years [interquartile range (IQR) 0.80-3.74].

Surgical tumor samples were fixed in formalin for more than 24 hours, embedded in paraffin, and stored in the archives of the Department of Pathology. All specimens underwent reevaluation by an experienced pathologist. Representative tumor areas were marked on hematoxylin eosin (HE) slides. Thereafter, TMA blocks including three 1.0-mm-diameter punches of tumor tissue were constructed [28,29].

Our second cohort consisted of 20 patients with pancreatic adenocarcinoma with pre- or postoperative FDG-PET scans. Clinical data and tissue samples were supplied by the Auria 
Biobank (Turku, Finland). These samples were whole-tissue slides and were analyzed separately from the Helsinki cohort.

\section{Immunohistochemistry}

The TMA blocks were cut into 4- $\mu$ m-thick sections, fixed on slides, and dried for 12 to 24 hours at 370C. Sections were subsequently deparaffinized in xylene and rehydrated through graded ethanol and distilled water. Immunohistochemistry for both TMA and whole-tissue samples was completed in a similar fashion. For antigen retrieval, the slides were treated with Tris-HCl (pH 8.5) in a PreTreatment module (LabVision Corp.) for 20 minutes at 980C. The staining of sections took place in an Autostainer 480 (LabVision) with an antihuman TKTL1 antibody (Rida Pentocheck IHC, Clone JFC12T10, R-Biopharm AG) diluted to 1:200 with Dako REAL Antibody Diluent S2022 (Dako). The primary antibody was kept on glasses overnight (O/N) followed by 30-minute incubation with the secondary peroxide-conjugated rabbit/mouse ENV (K5007) Dako REAL Envision/HRP antibody (Dako). The slides were finally visualized using the Dako REAL DAB+Chromogen kept on glasses for 10 minutes. Between each step in the staining procedure, slides were washed with PBS-0.04\% Tween20. The slides were counterstained using Meyer's hematoxylin, washed in tap water for 10 minutes, and finally mounted in an aqueous mounting medium (Aquamount, BHD). We used a gastric and a colon cancer specimen known to be TKTL1-positive as the positive control in each staining.

\section{Scoring}

Samples were scored independently by KA and JH. Cytoplasmic positivity of ductal epithelial cells was scored on a four-grade scale, where the absence of staining was scored as 0 , mild staining as 1 , moderate staining as 2 , and strong staining as 3 . The highest score out of three was selected to represent the tumor, since high TKTL1 appears to associate with poor survival 
in other gastrointestinal cancers. Samples with no tumor tissue or with too few cells for adequate evaluation were excluded. Samples receiving different scores from the two researchers were discussed in order to reach consensus. For analysis, samples were regrouped into two groups: low expression (scores 0 and 1) and high expression (scores 2 and 3).

\section{Statistical analysis}

We assessed the associations between TKTL1 and clinicopathological variables using the chisquare test. Disease-specific survival (DSS) was calculated from the date of surgery through the date of death from pancreatic adenocarcinoma or until the end of follow-up. We constructed survival curves based on the Kaplan-Meier method and compared them using the log-rank test. For the univariate and multivariate survival analyses, the Cox proportional hazard model entered the following covariates: age, gender, grade, TNM grade, tumor location (head, body, or tail of the pancreas), perineural and perivascular invasion, and TKTL1 expression. TNM grade, grade, histological type, and TKTL1 expression were entered as categorical covariates. We considered $\mathrm{p}<0.05$ statistically significant. All statistical analyses were performed using IBM’s SPSS Statistics for Mac, version 25.0 (IBM Corporation).

The Surgical Ethics Committee of Helsinki University Hospital (Dnro HUS 226/E6/06, extension TMK02 §66 17.4.2013) and the National Supervisory Authority for Welfare and Health (Valvira Dnro 10041/06.01.03.01/2012) approved the study protocol and granted us license to study the archived tissue samples without requiring specific individual consent.

\section{RESULTS}




\section{Immunohistochemistry}

We analyzed the cytoplasmic TKTL1 staining of tumor cells. In two patients, all TMA spots lacked tumor tissue for adequate evaluation and were excluded. Thus, 166 of 168 patients were included in the analyses. Among these, 13 patients $(7.8 \%)$ had a score of $0,80(48.2 \%)$ a score of 1, $57(34.3 \%)$ a score of 2, and $16(9.6 \%)$ a score of 3 (Fig. 1). For the subsequent analysis, scores 0 and 1 were combined to indicate negative staining and scores 2 and 3 represented positive staining.

The patient series from the Auria Biobank $(n=20)$ was too small for statistical analyses. Among these, 6 had disseminated disease and did not undergo pancreatic resection. The remaining 14 patients underwent surgery, either a pancreaticoduodenectomy (11 patients), pancreatic tail resection ( 2 patients), or removal of the entire pancreas ( 1 patient). There were 2 patients with a score of 0,7 patients with a score of 1,3 patients with a score of 2 , and 2 patients with a score of 3. Furthermore, 13 patients had an FDG-PET-CT prior to biopsy or surgery. Among these, 11 exhibited an increased FDG uptake around the pancreatic tumor; surprisingly, those two patients who did not, had unresectable disease.

\section{Associations}

We found no associations between the TKTL1 score and the covariates entered: age, gender, TNM classification, stage, or perivascular invasion (Table 1) nor between grade, American Society of Anesthesiologists (ASA) class, or perineural invasion (data not shown).

\section{Survival}

DSS at 5 years among all patients was 21.3\% [95\% confidence interval (CI) 14.6-28.0]. DSS was $17.3 \%$ (95\% CI 9.5-25.1) among the low expression group and $28.0 \%$ (95\% CI 16.4- 
39.6) among the high expression group, although this was not statistically significant ( $p=$

0.123; Fig. 2). In the univariate analysis, patients with perivascular invasion experienced a better survival than those with no perivascular invasion [hazard ratio (HR) $0.55,95 \% \mathrm{CI}$ 0.37-0.81; Table 3].

In the subgroup analysis, a high TKTL1 score marked a better prognosis among patients over $65(p=0.012$; Fig. 3a) as well as among patients with advanced disease: TNM class M1 ( $p=$ 0.018), perivascular invasion ( $p=0.008$; Fig. $3 b)$, and stage IV disease $(p=0.027)$ (Table 2$)$.

In the multivariate survival analysis, perivascular invasion, tumor location, and the TKTL1 score represented independent risk factors. Patients with a high TKTL1 expression exhibited a better prognosis than those with a low TKTL1 expression (HR 0.61, 95\% CI 0.38-1.00; Table 3).

\section{DISCUSSION}

We previously showed that a high TKTL1 expression in cancer cells correlates with a poor prognosis in gastric and colorectal cancer. TKTL1 and its prognostic value in PDAC have, to the best of our knowledge, not previously been examined. Here, we show in a rather large patient cohort that the prognostic value of TKTL1 in PDAC remains less clear compared to other cancers studied. However, in certain subgroups, a high TKTL1 expression served as a marker of better prognosis. This represents a slightly confusing finding since previous studies showed that a high TKTL1 expression typically associates with a poor prognosis. The difference between various cancers is difficult to explain. It is possible that the overall aggressive nature of PDAC in some way renders it different compared to other cancers. 
In a previous study, TKTL1 could be detected using the epitope detection in monocytes (EDIM) blood test in all 34 pancreatic cancer patients studied, whereas a healthy control group exhibited a negative EDIM TKTL1 score [30]. Circulating TKTL1 is, however, not specific to pancreatic cancer, but also accompanies colorectal and biliary cancer. In another study, transketolase, TKTL1, and TKTL2 all expressed in all pancreatic cancer cell lines tested. The mRNA levels of transketolase were significantly higher than those for TKTL1 and TKTL2 [31]. Normal pancreatic ductal cells express transketolase, whereas cancer cells exhibit higher TKTL1 levels. In our material, TKTL1 was expressed in most tumors ( $n=153$ or 92.2\%).

In our previous studies, TKTL1 tissue expression associated with poor survival in colorectal and gastric cancer $[6,32]$. Other groups have reported similar results in other cancer types, such as urothelial, non-small cell lung cancer, oral squamous cell carcinoma, and tumors of the ocular adnexa $[7,10,15,16]$.

TKTL1 appears to play a contradictory role in PDAC. Tumors invading the celiac axis, common hepatic, or superior mesenteric arteries (T4), and those with distant metastasis (M1) carry a poor prognosis. Our patient cohort was carefully selected with patients operated on with a curative intent, whereby only a few presented with advanced disease. Interestingly, in this very small subgroup of patients with pT4 or c/pM1, a high TKTL1 expression predicted a better prognosis. This tendency was similar across the entire cohort, although this finding was not significant. We, thus, must exercise caution in drawing conclusions based on this result. A similar effect was, however, seen in Diaz-Moralli's work on metastasized colorectal cancer, where TKTL1 expression in the primary tumor diminished in stage IV disease when examining expression levels using computational image analysis [33]. One possible 
explanation for this is that TKTL1 is necessary for tumor progression, but becomes unnecessary when the tumor progresses and metastasizes. Alternatively, tumors that do not strongly express TKTL1 are no longer able to grow locally, but have metastasized instead. Because PDAC is often advanced at the time of diagnosis, this may explain why TKTL1 is not a prognostic marker in this cancer type.

PET-CT represents a valuable diagnostic tool, but its value in the staging of PDAC remains vague at best [34]. In our small series, 18F-FDG-PET was positive in all of the PDAC surgical patients who underwent a preoperative scan. The patient series received from the Auria Biobank was too small to allow for adequate statistical analyses. We could, however, see that 18F-FDG-PET-CT was positive both for patients with a low and a high TKTL1 expression. A positive postoperative FDG-PET-CT signaled either a R1 resection or disease progression and was, thus, a sign of poor prognosis (data not shown). Using PET-CT in planning treatment for PDAC has thus far been limited to those with ambiguous CT scan findings and to those with a negative CT finding, but a rising tumor marker level. Unfortunately, the patient material with tissue specimens and PET results available for this study was too small to draw definitive conclusions. However, the associations between the tissue TKTL1 expression and glucose metabolism tumor markers, as well as the 18F-FDG-PET findings, remain interesting and require further exploration.

To conclude, here we show that TKTL1 cannot serve as a prognostic marker in pancreatic ductal adenocarcinoma with the exception of elderly patients and those with advanced disease. The correlation between TKTL1 and 18-FDG-PET-CT needs further study. The larger question remaining is how we can better treat our patients with PDAC. 


\section{ACKNOWLEDGEMENTS}

We thank Päivi Peltokangas and Pia Saarinen for their exceptional technical assistance. This study benefitted from samples and data from the Auria Biobank, Turku, Finland.

\section{STATEMENT OF ETHICS}

The Surgical Ethics Committee of Helsinki University Hospital (Dnro HUS 226/E6/06, extension TMK02 $\$ 66$ 17.4.2013) and the National Supervisory Authority for Welfare and Health (Valvira Dnro 10041/06.01.03.01/2012) approved the study protocol and granted us license to study the archived tissue samples without requiring specific individual consent.

\section{DISCLOSURE STATEMENT}

The authors have no conflicts of interest to declare.

\section{FUNDING SOURCES}

This work was financially supported by Finska Läkaresällskapet (KA, CB, CH), the K. Albin Johansson Foundation (KA, CB), the Finnish Cancer Foundation (HS), the Finnish State Subsidy Fund (HS), the Sigrid Jusélius Foundation (HS, CH) and Medicinska Understödsföreningen Liv och Hälsa $(\mathrm{CH})$.

\section{AUTHOR CONTRIBUTIONS}

The histological scoring was done by KA and JH, data collection by KS, HS and SK, statistical analyses by KA and CB, and study design by $\mathrm{CB}$ and $\mathrm{CH}$. All authors contributed to writing of the manuscript. 


\section{REFERENCES}

1. Bray F, Ferlay J, Soerjomataram I, Siegel RL, Torre LA, Jemal A. Global Cancer Statistics 2018: GLOBOCAN Estimates of Incidence and Mortality Worldwide for 36 Cancers in 185 Countries. CA Cancer J Clin. 2018 Sep 12;49(suppl 8):509.

2. Ettrich TJ, Berger AW, Perkhofer L, Daum S, König A, Dickhut A, et al. Neoadjuvant plus adjuvant or only adjuvant nab-paclitaxel plus gemcitabine for resectable pancreatic cancer - the NEONAX trial (AIO-PAK-0313), a prospective, randomized, controlled, phase II study of the AIO pancreatic cancer group. BMC Cancer. BioMed Central; 2018 Dec 29;18(1):1298.

3. Versteijne E, Suker M, Groothuis K, Akkermans-Vogelaar JM, Besselink MG, Bonsing BA, et al. Preoperative Chemoradiotherapy Versus Immediate Surgery for Resectable and Borderline Resectable Pancreatic Cancer: Results of the Dutch Randomized Phase III PREOPANC Trial. J Clin Oncol. 2020 Jun 1;38(16):1763-73.

4. Warburg O, Posener K, Negelein E. Über den Stoffwechsel der Carcinomzelle. Biochem Z. 1924;152:309-44.

5. Xu X, Hausen Zur A, Coy JF, Löchelt M. Transketolase-like protein 1 (TKTL1) is required for rapid cell growth and full viability of human tumor cells. International Journal of Cancer. 2009 Mar 15;124(6):1330-7.

6. Ahopelto K, Böckelman C, Hagström J, Koskensalo S, Haglund C. Transketolase-like protein 1 expression predicts poor prognosis in colorectal cancer. Cancer Biol Ther. Taylor \& Francis; 2016;17(2):163-8.

7. Langbein S, Zerilli M, Hausen Zur A, Staiger W, Rensch-Boschert K, Lukan N, et al. Expression of transketolase TKTL1 predicts colon and urothelial cancer patient survival: Warburg effect reinterpreted. Br J Cancer. 2006 Feb 27;94(4):578-85.

8. Schultz H, Kähler D, Branscheid D, Vollmer E, Zabel P, Goldmann T. TKTL1 is overexpressed in a large portion of non-small cell lung cancer specimens. Diagn Pathol. BioMed Central; 2008 Aug 12;3(1):35.

9. Sun W, Liu Y, Glazer CA, Shao C, Bhan S, Demokan S, et al. TKTL1 is activated by promoter hypomethylation and contributes to head and neck squamous cell carcinoma carcinogenesis through increased aerobic glycolysis and HIF1alpha stabilization. Clin Cancer Res. 2010 Feb 1;16(3):857-66.

10. Kayser G, Sienel W, Kubitz B, Mattern D, Stickeler E, Passlick B, et al. Poor outcome in primary non-small cell lung cancers is predicted by transketolase TKTL1 expression. Pathology. 2011 Dec;43(7):719-24. 
11. Krockenberger M, Engel JB, Schmidt M, Kohrenhagen N, Häusler SFM, Dombrowski Y, et al. Expression of transketolase-like 1 protein (TKTL1) in human endometrial cancer. Anticancer Res. 2010 May;30(5):1653-9.

12. Schmidt M, Voelker HU, Kapp M, Krockenberger M, Dietl J, Kammerer U. Glycolytic phenotype in breast cancer: activation of Akt, up-regulation of GLUT1, TKTL1 and down-regulation of M2PK. J Cancer Res Clin Oncol. Springer-Verlag; 2010 Feb;136(2):219-25.

13. Chen H, Yue J-X, Yang S-H, Ding H, Zhao R-W, Zhang S. Overexpression of transketolaselike gene 1 is associated with cell proliferation in uterine cervix cancer. J Exp Clin Cancer Res. 2009 Mar 30;28(1):43.

14. Schwaab J, Horisberger K, Ströbel P, Bohn B, Gencer D, Kähler G, et al. Expression of Transketolase like gene 1 (TKTL1) predicts disease-free survival in patients with locally advanced rectal cancer receiving neoadjuvant chemoradiotherapy. BMC Cancer. BioMed Central; 2011 Aug 19;11(1):363.

15. Lange CA, Tisch-Rottensteiner J, Böhringer D, Martin G, Schwartzkopff J, Auw-Haedrich C. Enhanced TKTL1 Expression in Malignant Tumors of the Ocular Adnexa Predicts Clinical Outcome. Ophthalmology. 2012 Sep;119(9):1924-9.

16. Grimm M, Munz A, Teriete P, Nadtotschi T, Reinert S. GLUT-1+/TKTL1+ coexpression predicts poor outcome in oral squamous cell carcinoma. Oral Surgery, Oral Medicine, Oral Pathology and Oral Radiology. 2014 Jun;117(6):743-53.

17. Bares R, Klever P, Hauptmann S, Hellwig D, Fass J, Cremerius U, et al. F-18 fluorodeoxyglucose PET in vivo evaluation of pancreatic glucose metabolism for detection of pancreatic cancer. Radiology. 1994 Jul;192(1):79-86.

18. Burge ME, O'Rourke N, Cavallucci D, Bryant R, Francesconi A, Houston K, et al. A prospective study of the impact of fluorodeoxyglucose positron emission tomography with concurrent non-contrast CT scanning on the management of operable pancreatic and peri-ampullary cancers. HPB (Oxford). 2015 Jul;17(7):624-31.

19. Wang X-Y, Yang F, Jin C, Fu D-L. Utility of PET/CT in diagnosis, staging, assessment of resectability and metabolic response of pancreatic cancer. World J Gastroenterol. 2014 Nov 14;20(42):15580-9.

20. Jha P, Bijan B. PET/CT for Pancreatic Malignancy: Potential and Pitfalls. J Nucl Med Technol. Society of Nuclear Medicine; 2015 Jun;43(2):92-7.

21. Ruf J, Lopez Hänninen E, Oettle H, Plotkin M, Pelzer U, Stroszczynski C, et al. Detection of recurrent pancreatic cancer: Comparison of FDG-PET with CT/MRI. Pancreatology. 2005 Apr 22;5(2-3):266-72.

22. Buck AC, Schirrmeister HH, Guhlmann CA, Diederichs CG, Shen C, Buchmann I, et al. Ki67 immunostaining in pancreatic cancer and chronic active pancreatitis: does in vivo FDG uptake correlate with proliferative activity? J Nucl Med. 2001 May;42(5):721-5. 
23. Sperti C, Pasquali C, Chierichetti F, Decet D, Ferronato A, Pedrazzoli S. 18-FDG pet as factor in predicting survival of patients with pancreatic carcinoma. Gastroenterology. Elsevier; 2003 Apr 1;124(4):A787.

24. Epelbaum R, Frenkel A, Haddad R, Sikorski N, Strauss LG, Israel O, et al. Tumor aggressiveness and patient outcome in cancer of the pancreas assessed by dynamic 18F-FDG PET/CT. J Nucl Med. Society of Nuclear Medicine; 2013 Jan;54(1):12-8.

25. Tempero MA, Malafa MP, Al-Hawary M, Asbun H, Bain A, Behrman SW, et al. Pancreatic Adenocarcinoma, Version 2.2017, NCCN Clinical Practice Guidelines in Oncology. Vol. 15, Journal of the National Comprehensive Cancer Network : JNCCN. 2017. pp. 1028-61.

26. Tempero MA. NCCN Guidelines Updates: Pancreatic Cancer. J Natl Compr Canc Netw. 2019 May 1;17(5.5):603-5.

27. Ducreux M, Cuhna AS, Caramella C, Hollebecque A, Burtin P, Goéré D, et al. Cancer of the pancreas: ESMO Clinical Practice Guidelines for diagnosis, treatment and follow-up. Vol. 26 Suppl 5, Annals of oncology : official journal of the European Society for Medical Oncology. 2015. pp. v56-68.

28. Kononen J, Bubendorf L, Kallioniemi A, Bärlund M, Schraml P, Leighton S, et al. Tissue microarrays for high-throughput molecular profiling of tumor specimens. Nat Med. 1998 Jul;4(7):844-7.

29. Kallioniemi OP, Wagner U, Kononen J, Sauter G. Tissue microarray technology for highthroughput molecular profiling of cancer. Hum Mol Genet. 2001 Apr;10(7):657-62.

30. Saman S, Stagno MJ, Warmann SW, Malek NP, Plentz RR, Schmid E. Biomarkers Apo10 and TKTL1: Epitope-detection in monocytes (EDIM) as a new diagnostic approach for cholangiocellular, pancreatic and colorectal carcinoma. Cancer Biomark. IOS Press; 2020;27(1):129-37.

31. Liu H, Huang D, McArthur DL, Boros LG, Nissen N, Heaney AP. Fructose induces transketolase flux to promote pancreatic cancer growth. Cancer Res. 2010 Aug 1;70(15):6368-76.

32. Ahopelto K, Laitinen A, Hagström J, Böckelman C, Haglund C. Transketolase-Like Protein 1 and Glucose Transporter 1 in Gastric Cancer. Oncology. Karger Publishers; 2020 May 20;98(9):1-10.

33. Diaz-Moralli S, Tarrado-Castellarnau M, Alenda C, Castells A, Cascante M. Transketolaselike 1 expression is modulated during colorectal cancer progression and metastasis formation. Lisanti M, editor. PLoS ONE. Public Library of Science; 2011;6(9):e25323.

34. Wang Z, Chen J-Q, Liu J-L, Qin X-G, Huang Y. FDG-PET in diagnosis, staging and prognosis of pancreatic carcinoma: a meta-analysis. World J Gastroenterol. Baishideng Publishing Group Inc; 2013 Aug 7;19(29):4808-17. 


\section{FIGURE LEGENDS}

Fig. 1. TKTL1 immunohistochemical staining of PDAC samples. Cytoplasmic intensity was scored along a four-grade scale. A) negative, B) mild, C) moderate, and D) strong. Original magnification: 400x.

Fig. 2. Disease-specific survival in PDAC according to TKTL1 expression. No significant difference between the low and high expression groups was found ( $p=0.123$ ).

Fig. 3. A high TKTL1 expression served as a marker of a better prognosis in a) patients older than 65 years $(p=0.012)$ and $b)$ patients with perivascular invasion $(p=0.008)$. 


\section{Figure 2}

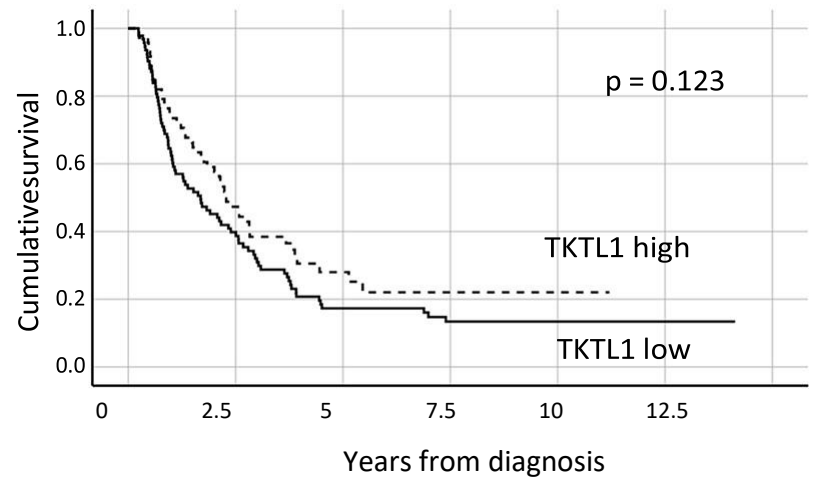

\section{Patients at risk}

TKTL1 high expression: 73

93

10

2

TKTL1 low expression:

15

5 


\section{Figure 3}

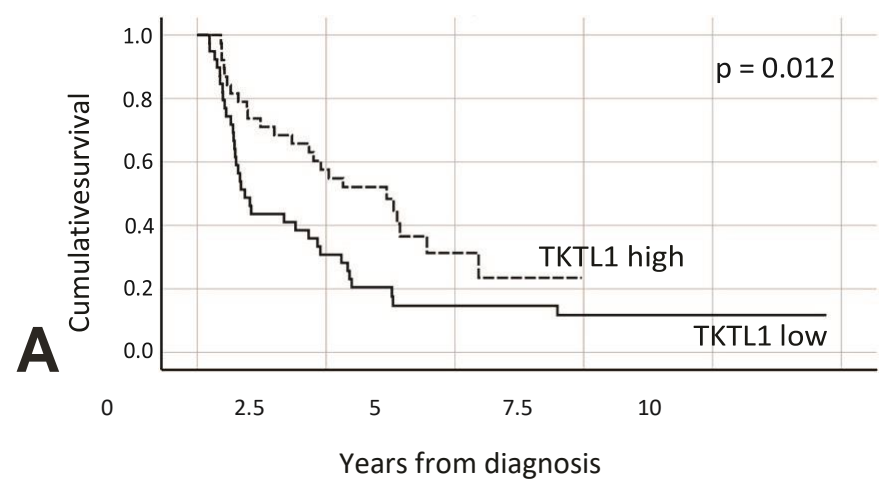

Patients at risk

TKTL1 high expression: $\quad 39 \quad 21 \quad 5 \quad 200$

$\begin{array}{llllll}\text { TKTL1 low expression: } & 39 & 12 & 5 & 4 & 1\end{array}$

\section{B}

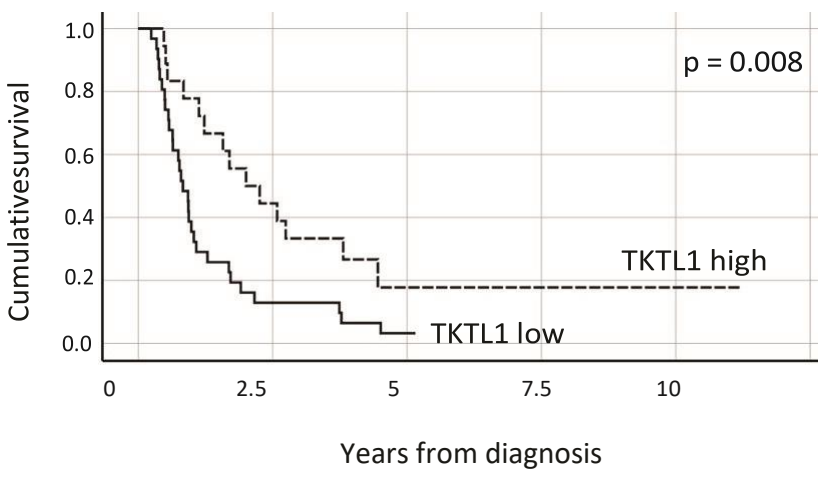

Patients at risk

$\begin{array}{llllll}\text { TKTL1 high expression: } & 18 & 8 & 1 & 1 & 0 \\ \text { TKTL1 low expression: } & 31 & 4 & 1 & 0 & 0\end{array}$


Table 1. Associations between TKTL1 and clinicopathological data in PDAC.

\begin{tabular}{|c|c|c|c|c|c|c|}
\hline \multicolumn{2}{|c|}{ Clinicopathological } & \multicolumn{2}{|c|}{$\begin{array}{l}\text { TKTL-1 low }(0-1) \\
\text { expression }\end{array}$} & \multicolumn{2}{|c|}{$\begin{array}{l}\text { TKTL-1 high (2-3) } \\
\text { expression }\end{array}$} & \multirow[b]{2}{*}{$P$-value* } \\
\hline variable & $n$ & $n$ & $\%$ & $n$ & $\%$ & \\
\hline
\end{tabular}


Age, years

$$
\begin{aligned}
& <65 \\
& \geq 65
\end{aligned}
$$

\section{Gender}

$\begin{array}{llllll}88 & 54 & 61.4 & 34 & 38.6 & 0.526 \\ 78 & 39 & 50.0 & 39 & 50.0 & \end{array}$

\section{Male}

Female

$\begin{array}{llllll}91 & 53 & 58.2 & 38 & 41.8 & \\ 75 & 40 & 53.3 & 35 & 46.7 & 0.591\end{array}$

\section{TNM Classification}

\section{T1}

$\mathrm{T} 2$

T3

$\mathrm{T} 4$

NO

N1

$\mathrm{N} 2$

M0

M1

\section{Stage}

$\begin{array}{cccccc}11 & 5 & 45.5 & 6 & 54.5 & \\ 42 & 27 & 64.3 & 15 & 35.7 & 0.573 \\ 106 & 57 & 53.8 & 49 & 46.2 & \\ 5 & 3 & 60.0 & 2 & 40.0 & \\ 48 & 25 & 52.1 & 23 & 47.9 & 1.000 \\ 116 & 66 & 56.9 & 50 & 43.1 & \\ & & & & & \\ 157 & 87 & 55.4 & 70 & 44.6 & \\ 7 & 4 & 57.1 & 3 & 42.9 & 0.774\end{array}$

IA

$$
\text { IB }
$$

IIA

IIB

III

IV

\section{Perivascular invasion}

$$
\text { Yes }
$$

$$
\text { No }
$$

\section{Location}

$\begin{array}{cc}9 & 3 \\ 17 & \\ 20 & 12 \\ 105 & 61 \\ 3 & 2 \\ 8 & \end{array}$

3
9
12
61
2
4

\section{3}

52.9

60.0

58.1

66.7

50.0

$\begin{array}{ll}49 & 31 \\ 88 & 44\end{array}$

63.3

50.0

18

44

36.7

50.0

0.231

Head

Body

Tail

Whole pancreas

$\begin{array}{ccccc}122 & 75 & 61.5 & 47 & 38.5 \\ 11 & 7 & 63.6 & 4 & 36.4 \\ 9 & 3 & 33.3 & 6 & 66.7 \\ 1 & 0 & 0 & 1 & 100.0\end{array}$

Abbreviations: TKTL1 = transketolase-like protein 1

Table 2. Kaplan-Meier analysis for disease-specific survival stratified for subgroups of pancreas. 


\section{Age, years}

$<=65$

$>65$

$19.9(11.3-28.5)$
$22.8(12.6-33.0)$

$19.3(8.7-29.9)$

$22.3(7.8-36.8)$

0.739

$>65$

\section{Gender}

Male

Female

18.9 (10.1-27.7)

15.8 (5.8-25.8)

$29.4(12.7-46,1)$

0.521

23.5 (13.5-33.5)

19.7 (7.4-32.0)

28.0 (12.3-43.7)

0.147

\section{Stage *}

IA

IB

IIA

IIB

III

IV

\section{Perivascular spreading}

yes

no

\section{Perineural spreading}

yes

no

20.5 (12.7-28.3)

14.3 (5.7-22.9)

30.3 (16.0-44.6)

0.070

$22.4(6.1-38.7)$

$15.0(-4.0-34.0)$

28.0 (2.9-53.1)

0.304

\section{Location}

head

body

tail

$\begin{array}{llll}18.8(11.5-26.1) & 19.6(10.6-28.6) & 18.3(5.76-30.8) & 0.895 \\ 0 & 0 & 0 & 0.140 \\ 37.0(0.3-73.7) & 33.3(0-86.6) & 44.4(1.0-87.9) & 0.177\end{array}$


Table 3. Cox regression analysis for DSS of PDAC patients.

\begin{tabular}{|c|c|c|c|c|c|c|}
\hline \multirow[b]{2}{*}{ Variable } & \multicolumn{3}{|c|}{ Univariable survival analysis } & \multicolumn{3}{|c|}{ Multivariable survival analysis } \\
\hline & \multicolumn{2}{|c|}{ Hazard ratio $95 \% \mathrm{CI}$} & \multirow[t]{2}{*}{ p-value } & \multicolumn{2}{|c|}{ Hazard ratio $95 \% \mathrm{CI}$} & \multirow{2}{*}{ p-value } \\
\hline Age, years & & & & & & \\
\hline$<=65$ & 1.00 & & & 1.00 & & \\
\hline$>65$ & 0.99 & $0.70-1.40$ & 0.959 & 0.86 & $0.55-1.36$ & 0.526 \\
\hline \multicolumn{7}{|l|}{ Gender } \\
\hline Male & 1.00 & & & 1.00 & & \\
\hline Female & 0.978 & $0-69-1.38$ & 0.899 & 1.13 & $0.73-1.74$ & 0.583 \\
\hline \multicolumn{7}{|l|}{ Stage } \\
\hline IA & 1.00 & & & 1.00 & & \\
\hline IB & 0.66 & $0.24-1.82$ & 0.417 & 0.91 & $0.19-4.29$ & 0.904 \\
\hline IIA & 1.11 & $0.43-1.88$ & 0.828 & 0.54 & $1.60-1.83$ & 0.324 \\
\hline IIB & 1.58 & $0.69-3.61$ & 0.283 & 0.58 & $0.19-1.83$ & 0.354 \\
\hline III & 3.92 & $0.97-15.94$ & 0.056 & 0.83 & $0.30-2.27$ & 0.716 \\
\hline IV & 1.89 & $0.63-5.65$ & 0.254 & 1.82 & $0.41-8.18$ & 0.432 \\
\hline \multicolumn{7}{|c|}{ Perivascular invasion } \\
\hline yes & 1.00 & & & 1.00 & & \\
\hline no & 0.55 & $0.37-0.81$ & 0.002 & 0.63 & $0.39-1.00$ & 0.048 \\
\hline \multicolumn{7}{|c|}{ Perineural invasion } \\
\hline yes & 1.00 & & & 1.00 & & \\
\hline no & 0.74 & $0.47-1.15$ & 0.182 & 0.89 & $0.52-1.54$ & 0.679 \\
\hline \multicolumn{7}{|l|}{ Location } \\
\hline head & 1.00 & & & 1.00 & & \\
\hline body & 0.01 & $0-0.15$ & 0.001 & 0.003 & $0-0.07$ & $<0.001$ \\
\hline tail & 0.01 & $0-0.16$ & 0.001 & 0.004 & $0-0.07$ & $<0.001$ \\
\hline whole pancreas & 0.02 & $0-0.18$ & 0.001 & 0.005 & $0-0.09$ & $<0.001$ \\
\hline \multicolumn{7}{|l|}{ TKTL1 } \\
\hline low & 1.00 & & & 1.00 & & \\
\hline
\end{tabular}


